\title{
OPTICAL DETECTION OF CYCLOTRON RESONANCE IN SERPENTINE SUPERLATTICE QUANTUM-WIRE ARRAYS
}

\author{
K. ŚWIĄTEK* , H. WEMAN \\ Department of Physics and Measurement Technology, IFM/FOA, Linköping University \\ 58183 Linköping, Sweden
}

M.S. Miller, P.M. Petroff and J.L. Merz

Center for Quantized Electronic Structures, QUEST, University of California

Santa Barbara, CA 93 106, USA

\begin{abstract}
We report low-temperature studies of microwave-induced cyclotron resonance of photo-generated carriers in $(\mathrm{Al}, \mathrm{Ga}) \mathrm{As}$ serpentine superlattice quantum-wire arrays. The geometric size of the parabolic-crescent cross-section of the quantum wires was of the order of $100 \AA \times 50 \AA$, depending on the angle of the vicinal substrate and the amount of parabolic curvature. Comparing the obtained spectra, we estimate the relative degree of carrier confinement in the ordered AlGaAs structure.
\end{abstract}

PACS numbers: 78.66.Fd, 76.40.+b, 71.25.Jd

\section{Introduction}

The confinement of electrons and holes to low-dimensional structures holds the promise of interesting physical properties, as well as potential device applications. The optical properties of one-dimensional (1D) quantum wires are expected to show enhanced oscillator strengths, non-linear effects and increased excitonic binding energies compared to the quantum well case, due to the peaked nature of the 1D density of states [1]. The growth of $(\mathrm{Al}, \mathrm{Ga}) \mathrm{As}$ serpentine superlattices (SSLs) on vicinal substrates has recently provided a promising way to fabricate quantum wire arrays with a lateral width of just a few $\mathrm{nm}$ [2]. In this paper we study the influence of external microwave and magnetic fields on the behavior of photo-excited carriers confined in the SSL region.

*Permanent address: Institute of Physics, Polish Academy of Sciences, Al. Lotników 32/46, 02-668 Warszawa, Poland. 


\section{Experimental details}

The SSL structures were grown by an alternating molecular beam epitaxy technique on $0.5^{\circ}$ and $2^{\circ}$ misoriented vicinal (100) GaAs substrates, corresponding to a stepped surface with a terrace width of $324 \AA$ and $81 \AA$, respectively. The parabolic curvature is determined by the misorientation angle and the linear ramping of the per-cycle coverage per layer thickness. The ramping constant was $125 \mathrm{~nm}$, with a total SSL layer thickness of $340 \AA$. The vertical barriers were nominally intended to cover $1 / 2$ of a step with a digital $\mathrm{Al}_{x} \mathrm{Ga}_{1-x} \mathrm{As}$ alloy composition of $x=20 \%$. The SSL layer is clad above and below with thick AlAs layer to confine the photo-excited carriers to the SSL region (Fig. 1, inset).

As a reference to the SSL samples we also grew an alloy-well model sample which incorporates the same buffering and cladding layers, but replaces the SSL with an alloy-well structure of the same thickness and average composition $\left(x_{\text {average }}=10 \%\right)$. For optically detected cyclotron resonance (ODCR) and photoluminescence (PL) experiments we used a modified Bruker 200D-SRC X-band ESR spectrometer equipped with a microwave cavity with optical access in all directions. The samples were excited with an $\mathrm{Ar}^{+}$laser ( $5145 \AA$ line). The resulting PL emissions were monitored by a cooled Ge detector via a $0.25 \mathrm{~m}$ Jobin-Yvon monochromator. All measurements were performed at $4 \mathrm{~K}$.

\section{Results and discussion}

In Fig. 1 the PL spectrum of the $2^{\circ}$-SSL sample is shown (upper curve). It contains two peaks identified as the heavy-hole exciton recombining in the active wire (left side) and well (right side) region. The main spectral difference between the alloy-well and the SSL peaks is the wider linewidths in case of the SSL samples. This is most likely due to inhomogeneous fluctuations in the serpentine potential due to both compositional and geometrical variations [3]. The peak position of the SSL emission depends on the SSL geometry and the lateral composition modulation. Since Ga re-evaporates during the SSL growth, it is not possible to estimate the lateral confinement directly from the energy position of the PL peak in Fig. 1. Instead, the lateral confinement was estimated by measuring the linear polarization of the PL peak $[2,3]$. In this work we are interested to see if spatial confinement is able to influence or thwart the cyclotron resonance of photo-generated carriers in the SSL samples. The microwave-induced change, $\Delta I_{\mathbf{P L}}^{\mathrm{ODCR}}$, in the luminescence is shown in Fig. 1 (lower curve), and it can be clearly seen that the sign of the signal depends on the energy of emission being monitored. At the SSL luminescence peak the microwave-induced change amounts to a $2 \%$ increase on the high-energy part and $3 \%$ decrease on the low-energy part of the emission intensity, for $200 \mathrm{~mW}$ of microwave power $(\nu=9.2 \mathrm{GHz})$ incident in the cavity. It is a distinct indication of potential fluctuations present in the ordered $(\mathrm{Al}, \mathrm{Ga})$ As SSL structures.

Figure 2 presents, as an example, the ODCR spectra of the $2^{\circ}$-SSL sample for two different directions of the external magnetic field: parallel ( $x$-direction) and perpendicular ( $y$-direction) to the wire. The ODCR signal was obtained for the $743 \mathrm{~nm}$ emission, which corresponds to monitoring the minimum of the $\Delta I_{\mathrm{PL}}^{\mathrm{ODCR}}$ 


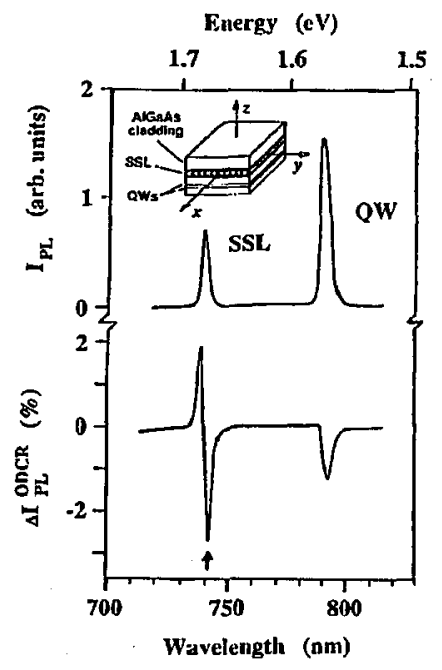

Fig. 1. The photoluminescence spectrum of the $2^{\circ}$-SSL quantum wire measured at $4 \mathrm{~K}$, with 514-nm Ar${ }^{+}$laser line excitation (upper curve). Microwave-induced change in the photoluminescence spectrum. (zero magnetic field, lower curve). Inset: schematic figure of the serpentine superlattice structure with the definition of the $x-, y$-, and $z$-axes.

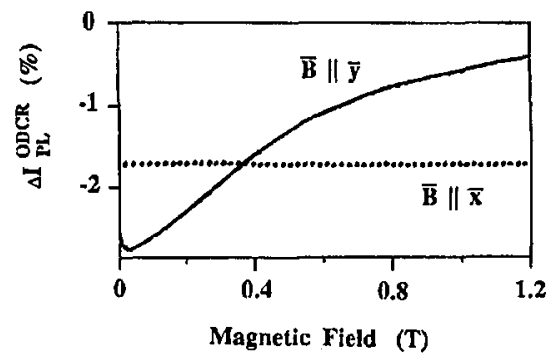

Fig. 2. The ODCR signal as a function of the applied magnetic field in the $x$ - and $y$-direction of the $2^{\circ}$-serpentine superlattice.

curve in Fig. 1 (see arrow). The cyclotron resonance (CR) induces a resonant decrease in this luminescence. A similar CR signal was also observed for the $738 \mathrm{~nm}$ emission, corresponding to monitoring the maximum of the $\Delta I_{\mathrm{PL}}^{\mathrm{ODCR}}$ curve in Fig. 1, but here the CR induces an increase in the luminescence. The resonant change amounts to $3 \%$ of the luminescence intensity.

Due to the fact that the resonance line is rather broad we can only estimate a longitudinal effective mass for the photo-generated carriers in the $2^{\circ}$-SSL wire of $(0.07 \pm 0.03) m_{0}$, which is comparable to the GaAs bulk effective mass for the electrons of $0.067 m_{0}$. We obtained the similar results for the $0.5^{\circ}-\mathrm{SSL}$ wire. The cyclotron orbit parameter at the highest applied magnetic field used $(1.2 \mathrm{~T})$ is $470 \AA$. This means that the cyclotron orbit is much larger than the geometrical di- 
mensions of the confinement, and in our experiments we are in the low-field regime. Therefore, the vertical and lateral electrostatic confinement should dominate over the magnetic field-induced confinement. Due to this fact we have not observed any trace of CR when the magnetic field was applied in the parallel direction ( $x$-direction) to the wire. Continuing our study, we plan to use higher magnetic fields and resonant excitation energy to achieve higher spectral resolution of the CR traces, as has been observed in ODCR measurements of bulk crystals $[4,5]$ and quantum wells [6].

\section{References}

[1] K. Kash, J. Lumin. 46, 69 (1990) and references therein.

[2] M.S. Miller, H. Weman, C.E. Pryor, M. Krishnamurthy, P.M. Petroff, H. Kroemer, J.L. Merz, Phys. Rev. Lelt. 68, 3464 (1992).

[3] H. Weman, M.S. Miller, C.E. Pryor, P.M. Petroff, H. Kroemer, J.L. Merz, Proc. SPIE's 1992 Conf. on "Quantum Well and Superlattice Physics IV", Somerset (USA) 1992, Vol. 1675, p. 120.

[4] P.G. Baranov, Yu.P. Veshchunov, R.A. Zhitnikov, N.G. Romanov, Yu.G. Shreter, Pis'ma Zh. Eksp. Teor. Fiz. 26, 369 (1977) [JETP Lett. 26, 249 (1977)].

[5] R. Romestain, C. Weisbuch, Phys. Rev. Lett. 45, 2067 (1980).

[6] B.C. Cavenett, E.J. Pakulis, Phys. Rev. B 32, 8449 (1985). 